Results. Adult studies: In short-term studies, TEM rates were comparable in patients treated with lurasidone monotherapy 20 $60 \mathrm{mg} / \mathrm{d}(3.7 \%)$ and $80-120 \mathrm{mg} / \mathrm{d}$ (1.9\%) vs. placebo (1.9\%). TEM rates were also comparable in patients treated with lurasidone 20$120 \mathrm{mg} / \mathrm{d}(1.1 \%)$ adjunctive to Li/VPA vs. placebo + Li/VPA (1.2\%). In the monotherapy study, significant reduction in YMRS score was observed at study endpoint for the $20-60 \mathrm{mg} / \mathrm{d}$ group compared to placebo $(-1.9$ vs. $-1.3 ; \mathrm{p}<0.05)$ with similar improvement relative to placebo in the $80-120 \mathrm{mg} / \mathrm{d}$ group. Change for YMRS score was comparable for lurasidone and placebo in the adjunctive study. In long-term studies, $1.3 \%$ of adult patients treated with lurasidone monotherapy $(n=316)$ met criteria for mania, and $3.8 \%$ of patients on adjunctive lurasidone therapy $(\mathrm{n}=497)$ met TEM criteria. Pediatric studies: TEM rates were comparable in patients treated with lurasidone vs. placebo $(1.7 \%$ vs. 2.3\%). LS mean reduction in symptoms of mania from baseline to week 6 was significantly greater for lurasidone vs. placebo on YMRS score $(-2.0$ vs. $-1.1 ; \mathrm{p}<0.05)$. Pediatric long-term studies: After two years of OL treatment with lurasidone, $5.2 \%$ of patients met TEM criteria. Mean change in YMRS total score from DB baseline to Month 24 continued to improve $(-2.0)$.

Conclusions. Short-term and long-term treatment with lurasidone demonstrated significant improvement in manic symptoms and was not associated with an increased risk of TEM in either adult or pediatric patient populations compared to rates reported in clinical populations of patients.

Funding. Sunovion Pharmaceuticals Inc.

\section{Long-Term Effectiveness of Lurasidone in Pediatric Bipolar Depression: Response, Remission and Recovery}

\section{Manpreet Singh, $\mathrm{MD}^{1}$, Michael Tocco, $\mathrm{PhD}^{2}$, Edward Schweizer, $\mathrm{MD}^{3}$ and Andrei Pikalov, MD, $\mathrm{PhD}^{2}$}

${ }^{1}$ Stanford University, Stanford Pediatric Mood Disorders Program, Stanford, CA, USA, ${ }^{2}$ Sunovion Pharmaceuticals Inc., Marlborough, MA, USA, and ${ }^{3}$ Paladin Consulting Group, Princeton, NJ, USA

Presenting Author: Michael Tocco response or remission in children and adolescents with bipolar depression followed over a two-year period.

Method. Patients 10-17 years with bipolar I depression who completed a 6-week double-blind (DB) study of lurasidone vs. placebo were eligible to enroll in a two-year, open-label (OL) extension study in which patients were continued on flexibly-dosed lurasidone (20-80 mg/d) or switched from placebo to lurasidone. Efficacy measures included the Children's Depression Rating Scale, Revised (CDRS-R) and the Clinical Global Impression, Bipolar Depression Severity scale (CGI-BP-S). Functioning was evaluated utilizing the Clinician-rated Children's Global Assessment Scale (CGAS) score, with a score $>70$ indicating no clinically meaningful functional impairment. Responder criteria were met if a patient achieved criteria $=50 \%$ reduction from $\mathrm{DB}$ baseline in the CDRS-R total score: remission criteria were met if a patient achieved a CDRS-R Total Score $=28$ and a YMRS total score $=8$ and CGI-BP-S depression score $=3$, and a patient was considered to have met recovery criteria if they achieved remission with a CGAS score $>70$. In addition, a more stringent outcome, sustained remission, was also analyzed, which required a patient to meet remission criteria for $=24$ consecutive weeks.

Results. A total of 306 patients completed the 6-week DB study and entered the extension study; 195 (63.7\%) patients completed one year of treatment and 168 (54.9\%) patients completed two years of treatment. Responder rates at OL baseline, one year, and two years were: $51.0 \%, 88.4 \%$ and $91.1 \%$, respectively; remission rates were $24.3 \%, 61.3 \%$, and $75.6 \%$, respectively; and recovery rates were $17.7 \%, 53.8 \%$, and $73.8 \%$. On a Pearson correlation analysis, there was a strong inverse relationship $(r=-0.71)$ between CDRS-R total score, and global functioning as measured by the CGAS. Sustained remission was achieved by $37.2 \%$ of patients at one year and $57 \%$ of patients after two years. Conclusions. In children and adolescents with bipolar depression, up to 2 years of treatment with lurasidone was associated with continued improvement in depressive symptoms, resulting in progressively higher rates of response, remission, recovery, and the more rigorously calculated outcome of sustained remission. Funding. Sunovion Pharmaceuticals Inc.

\title{
Safety and Effectiveness of SEP-363856 in Schizophrenia: Results of a 6-Month, Open-Label Extension Study
}

Christoph U. Correll, $\mathrm{MD}^{1}$, Kenneth S. Koblan, $\mathrm{PhD}^{2}$, Seth C. Hopkins, $\mathrm{PhD}^{2}$, Justine Kent, $\mathrm{MD}^{2}$, Hailong Cheng, $\mathrm{PhD}^{2}$, Robert Goldman, $\mathrm{PhD}^{2}$ and Antony Loebel, MD ${ }^{2}$

${ }^{1}$ The Zucker Hillside Hospital, Department of Psychiatry, Northwell Health, Glen Oaks, NY, USA; Hofstra Northwell School of Medicine, Department of Psychiatry and Molecular Medicine, Hempstead, NY, USA; and Charité Universitat Medizin, Department of Child and Adolescent Psychiatry, Berlin, Germany, and ${ }^{2}$ Sunovion Pharmaceuticals Inc., Marlborough, MA, USA

Presenting Author: Kenneth S. Koblan 


\section{Abstract}

Background. SEP-363856 is a novel psychotropic agent without dopamine D2 receptor occupancy. Although its mechanism of action has not been fully elucidated, preclinical data suggest that agonism at trace amine receptor 1 (TAAR1) and the serotonin 5-H1A receptor contributes to its efficacy. In a double-blind (DB), placebo-controlled study, SEP-363856 demonstrated significant efficacy in the treatment of an exacerbation of schizophrenia (Koblan et al, NEJM 2020; 82:1497-1506). We present results of a 6-month extension study whose aim was to evaluate the safety and effectiveness of longer-term treatment with SEP-363856.

Method. Patients with an acute exacerbation of schizophrenia who completed a 4-week, DB, placebo-controlled, flexible-dose (50 or $75 \mathrm{mg}$ ) study of SEP-363856 were given the option to enroll in an extension study in which they were treated, openlabel (OL), with flexible doses $(25 / 50 / 75 \mathrm{mg} / \mathrm{d})$ of SEP-363856 for 26-weeks. The primary outcomes were safety measures; effectiveness outcomes were secondary and included the PANSS total score and the Brief Negative Symptom Scale (BNSS) total score. Results. A total of 193 patients completed the 4-week DB study, and $156(80.8 \%)$ were dosed in the OL extension study and received at least one dose of SEP-363856 (safety population). Study completer rate was $66.9 \%$; reasons for discontinuation consisted of adverse event (11.5\%), withdrawal of consent (10.2\%), lack of efficacy (5.1\%), and other (6.4\%). 15 patients experienced an SAE: schizophrenia $(n=11)$; acute psychosis $(\mathrm{N}=1)$; uterine hemorrhage and suicidal ideation $(\mathrm{N}=1$ each); there were no deaths in the study. Individual AEs with an incidence $=2 \%$ were schizophrenia $(12.2 \%)$, headache $(11.5 \%)$, insomnia $(8.3 \%)$, anxiety $(5.1 \%)$, somnolence $(4.5 \%)$, nasopharyngitis (4.5\%), nausea (3.8\%), irritability (3.2\%), influenza (3.2\%), weight decreased $(3.2 \%)$, and prolactin increased $(2.6 \%)$. On movement scales, minimal mean change from OL-baseline to Week 26 occurred on the Barnes total score $(-0.1)$, AIMS total score $(0.0)$ and SAS score $(-0.1)$. Mean month 6 change from DB baseline in weight was $-0.3 \mathrm{~kg}$. No clinically meaningful median changes were observed at week 26 in metabolic laboratory parameters (total and LDL cholesterol, triglycerides, hemoglobin A1c) or in prolactin levels. During 6 months of OL treatment, one patient had an increase in QTcF $=60 \mathrm{msec}$; no patients had a QTcF interval $=480 \mathrm{msec}$. Treatment with SEP-363856 was associated with significant improvement from OL baseline to week 26 in PANSS total score $(-22.6)$ and BNSS total score $(-11.3)$.

Conclusion. Treatment with SEP-363856 was associated with continued improvement from open-label baseline in the PANSS total (-22.6) and BNSS total (-11.3) scores. The most frequently reported adverse events $(=5 \%)$ were schizophrenia, headache, insomnia and anxiety. SEP-363856 had minimal effects on weight, lipids, glycemic indices, prolactin, and was associated with minimal risk of extrapyramidal symptom.

Funding. Sunovion Pharmaceuticals Inc.

\section{Understanding the Evolving Continuing Medical Education Needs of Physicians Managing Patients with TD}

\author{
Shereta Wiley, $\mathrm{MPH}^{1}$, Wendy Cerenzia, $\mathrm{MS}^{1}$, \\ Sylvie Stacy, $M^{1}$, Chirag Shah, PharmD ${ }^{2}$, \\ Leslie Lundt, $\mathrm{MD}^{2}$ and Khody Farahmand, PharmD ${ }^{2}$
}

${ }^{1}$ CE Outcomes, LLC, Birmingham, AL, USA, and ${ }^{2}$ Neurocrine Biosciences, Inc., San Diego, CA, USA

Presenting Author: Shereta Wiley

\begin{abstract}
This study sought to understand the evolving continuing medical education (CME) needs of physicians managing patients with tardive dyskinesia (TD). A case-based survey was developed, and later updated, to assess current practice, knowledge, and attitudes of neurologists and psychiatrists in the management of patients with TD. The original and updated survey were fielded in May 2018 and March 2020, respectively, to US-practicing psychiatrists and neurologists. Results were obtained from 213 psychiatrists and 187 neurologists in 2018 and from 125 psychiatrists and 128 neurologists in 2020. Less than half of physicians in both 2018 and 2020 were able to correctly identify the prevalence of TD in patients on maintenance antipsychotics, with many underestimating reported prevalence. Respondents reported moderate familiarity with VMAT2 inhibitor therapies for TD, with self-reported familiarity increasing more among neurologists than psychiatrists since the 2018 study. Psychiatrists are more likely than neurologists to take responsibility for medical management of TD symptoms and antipsychotic medication adjustment. Despite recommendations from APA guidelines and AAN reviews, 15\% of physicians would use an anticholinergic to manage TD symptoms and only about half would opt for a VMAT2 inhibitor. There was a larger increase in VMAT inhibitor use between 2018 and 2020 among neurologists as compared to psychiatrists. The findings support the need for CME on TD focused toward specific provider groups. While both types of specialists would benefit from CME on the topic of TD epidemiology, there is an increased need for CME that includes treatment updates among psychiatrists.

Funding. Neurocrine Biosciences, Inc.
\end{abstract}

